\title{
Itinerant ferromagnetism in a Fermi gas with contact interaction: Magnetic properties in a dilute Hubbard model
}

\author{
Chia-Chen Chang $^{1}$, Shiwei Zhang ${ }^{1,2,3}$, and David M. Ceperley ${ }^{4}$ \\ ${ }^{1}$ Department of Physics, College of William and Mary, Williamsburg, VA 23187, USA \\ ${ }^{2}$ Institute of Physics, Chinese Academy of Sciences, Beijing 100190, China \\ ${ }^{3}$ Department of Physics, Renmin University of China, Beijing 100872, China \\ ${ }^{4}$ Department of Physics, University of Illinois at Urbana-Champaign, Urbana, IL 61801, USA
}

\begin{abstract}
Ground state properties of the repulsive Hubbard model on a cubic lattice are investigated by means of the auxiliary-field quantum Monte Carlo method. We focus on low-density systems with varying on-site interaction $U / t$, as a model relevant to recent experiments on itinerant ferromagnetism in a dilute Fermi gas with contact interaction. Twist-average boundary conditions are used to eliminate open-shell effects and large lattice sizes are studied to reduce finite-size effects. The sign problem is controlled by a generalized constrained path approximation. We find no ferromagnetic phase transition in this model. The ground-state correlations are consistent with those of a paramagnetic Fermi liquid.
\end{abstract}

PACS numbers: $71.10 . \mathrm{Fd}, 02.70 . \mathrm{Ss}$

Introduction - The study of ferromagnetism has a long history in physics. At the microscopic level, the formation of ferromagnetic order is a consequence of strong interactions. Heisenberg first pointed out that an exchange interaction that lowers the energy of a pair of parallel spins would favor ferromagnetism. However a localizedspin mechanism cannot be fully responsible for ferromagnetism in transition metals, for instance iron and nickel, where electrons are extended. Both the interactions and the delocalized nature of electrons have to be taken into account at a more fundamental level.

A generic description of itinerant ferromagnetism is given by the three-dimensional (3-D) Hubbard model [1]:

$$
H=-t \sum_{\langle i j\rangle \sigma}\left(c_{i \sigma}^{\dagger} c_{j \sigma}+\text { h.c. }\right)+U \sum_{i} n_{i \uparrow} n_{i \downarrow} .
$$

The operator $c_{i \sigma}^{\dagger}\left(c_{i \sigma}\right)$ creates (annihilates) an electron with spin $\sigma(\sigma=\uparrow, \downarrow), i$ enumerates the sites in an $N=L^{3}$ lattice, and $\langle i j\rangle$ denotes a sum of nearest neighbor pairs. The parameter $t$ is the nearest-neighbor hopping amplitude, and $U>0$ is the on-site interaction strength. The total density is $n=\left(N_{\uparrow}+N_{\downarrow}\right) / N$. This simple Hamiltonian contains both the itinerant character and local repulsion. However, because neither of the two terms alone favors ferromagnetic ordering, the magnetic correlations in the Hubbard model is not obvious.

The first evidence of ferromagnetism in the Hubbard model was discussed by Nagaoka [2] and by Thouless [3]. They showed that the ground state with a single hole in any finite bipartite lattice with $U \rightarrow \infty$ (and periodic boundary conditions) is fully polarized. Subsequent studies indicate that the stability of the state with more holes can depend on system size [4], and boundary conditions 5]. The critical doping for the onset of ferromagnetism is still an open question [4, 6, 7]. Away from infinite- $U$, the existence of ferromagnetism at non-zero density is less certain. Whether ferromagnetism is a generic property of the Hubbard model is still not answered.
Rapid experimental progress in cold atoms has opened a new avenue for exploring the physics of itinerant ferromagnetism. In a recent experiment aimed to simulate the Stoner Hamiltonian (i.e. spin $1 / 2$ fermions in continuous space interacting with a repulsive contact potential), a dilute gas of two hyperfine states of ${ }^{6} \mathrm{Li}$ atoms are tuned to interact via large positive scattering lengths. Signatures of ferromagnetic instability 8$]$ have generated a lot of theoretical interest [9 12 .

The Hubbard Hamiltonian in Eq. (1) gives a reasonable representation of the Stoner Hamiltonian on a lattice. As the density $n \rightarrow 0$ it seems clear that no ferromagnetism exists in the model in Eq. (10) 13], since the maximum scattering length is bounded by $\sim 1 / 3.173$ lattice spacing [14, 15]. However, at low but not zero density, the magnetic properties and the phase diagram of the 3-D Hubbard model are not clear. We address this question here by very accurate many-body simulations with the constrained path Monte Carlo (CPMC) method.

Method - The CPMC method [16 18] projects the many-body ground state $\left|\Psi_{0}\right\rangle$ from a trial wave function $\left|\Psi_{T}\right\rangle$ by repeated application of an imaginary-time propagator $e^{-\Delta \tau H}(\Delta \tau$ is the Trotter time step), provided that $\left|\Psi_{T}\right\rangle$ satisfies $\left\langle\Psi_{0} \mid \Psi_{T}\right\rangle \neq 0$. The propagator is decomposed into $e^{-\Delta \tau H} \approx e^{-\Delta \tau H_{1} / 2} e^{-\Delta \tau H_{2}} e^{-\Delta \tau H_{1} / 2}+$ $\mathcal{O}\left(\Delta \tau^{3}\right)$, where $H_{1}$ and $H_{2}$ are one- and two-body parts of $H$ respectively. The two-body part $e^{-\Delta \tau H_{2}}$ is further decoupled into a sum over one-body projectors in Ising fields 19]. This leads to a formally exact expression $e^{-\Delta \tau H}=\sum_{\{\mathbf{x}\}} P(\{\mathbf{x}\}) B(\{\mathbf{x}\})$, where $\{\mathbf{x}\}$ is a collection of $N$ Ising fields, $P(\{\mathbf{x}\})$ is their probability distribution, and $B(\{\mathbf{x}\})$ is a one-body projector. The multidimensional summation is carried out efficiently by importancesampled random walks with non-orthogonal Slater determinants (SDs), where the one-body projectors $B(\{\mathbf{x}\})$ propagate one SD into another.

The fermion sign problem is controlled approximately by the constrained path approximation. [16] The many- 
body ground state is given by $\left|\Psi_{0}\right\rangle=\sum_{\phi} w(\phi)|\phi\rangle$, where $|\phi\rangle$ are SDs sampled by the QMC, and their probability distribution determines the weight factors $w(\phi)$. Because the Schrödinger equation is linear, $\left|\Psi_{0}\right\rangle$ is degenerate with $-\left|\Psi_{0}\right\rangle$. In a random walk, the SDs can move back and forth between the two sets of solutions. The appearance of the two sets with opposite signs in the Monte Carlo samples is the origin of the sign problem. To control the problem, the walker is required to satisfy the constraint $\left\langle\Psi_{T} \mid \phi\right\rangle>0$ in the course of the random walk. This is the only approximation in our method. More formal discussions of the theoretical basis of the generalized constrained path approximation and benchmarks can be found elsewhere [16, 17]. In the Hubbard model, the energy at $U=4 t$ is typically within $<0.5 \%$ of the exact diagonalization result [18]. Extensive benchmarks of this approach for molecules and solids are in Refs. [20, 21].

The constrained path approximation is similar in spirit to the fixed-node approximation in the diffusion Monte Carlo (DMC) method [22, 23], which has been used for all recent simulation work on the problem of itinerant ferromagnetism in the Stoner model 10, 11, 24]. In fixednode DMC one uses a real-space trial function $\Psi_{T}(R)$ to determine the sign of the ground-state wave function. The random walks, which involve movements of electron coordinates $R$ (a $3\left(N_{\uparrow}+N_{\downarrow}\right)$-dimensional vector), are constrained to the region where $\Psi_{T}(R)>0$. Since, in CPMC the random walks take place in the space of SDs, where fermionic statistics are automatically maintained, the sign problem is reduced. As a result, the constrained path approximation is less sensitive to $\left|\Psi_{T}\right\rangle$ and typically has smaller systematic errors.

In this work we apply twist-averaged boundary conditions (TABCs) 25]. Under TABCs, the wave function gains a phase when electrons wind around the periodic boundary conditions: $\Psi\left(\ldots, \mathbf{r}_{j}+\mathbf{L}, \ldots\right)=$ $e^{i \widehat{\mathbf{L}} \cdot \boldsymbol{\Theta}} \Psi\left(\ldots, \mathbf{r}_{j}, \ldots\right)$, where $\widehat{\mathbf{L}}$ is the unit vector along $\mathbf{L}$, and $\boldsymbol{\Theta}=\left(\theta_{x}, \theta_{y}, \theta_{z}\right)$ are random twists over which we average. A simple generalization of the CPMC method can be made to handle the overall phase that arises from TABC [17, 18]. As an additional benchmark for the present work, we studied several low-density $L=4$ systems in detail. For example, at $U=16 t$ with $n=0.25$, the CPMC energy, averaged over $1000 \Theta$-points, agrees to better than $0.2 \%$ with exact diagonalization.

Energy - We first compare the ground-state energy of an unpolarized system $\left(N_{\uparrow}=N_{\downarrow}\right)$ with that of a fully polarized state at the same total density. The results are summarized in Fig. 1. Because electrons of the same spin do not interact, the energy of the fully polarized state, $e_{F M}$, is purely kinetic and does not depend on $U$. In mean-field (MF) theory, the energy of a system with $n_{\sigma}=N_{\sigma} / N$ (with $n=n_{\uparrow}+n_{\downarrow}$ ) is $e_{M F}(U, n)=$ $\left[e_{0}\left(n_{\uparrow}\right) n_{\uparrow}+e_{0}\left(n_{\downarrow}\right) n_{\downarrow}+U n_{\uparrow} n_{\downarrow}\right] / n$, where $e_{0}\left(n_{\sigma}\right)$ is the energy of the fully polarized system at density $n_{\sigma}$. At
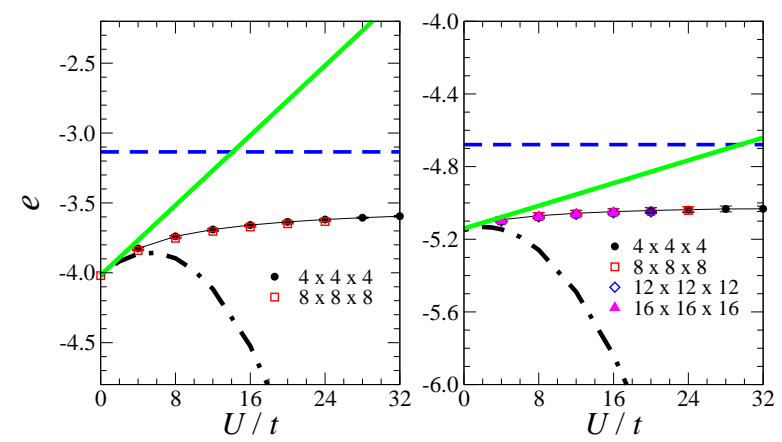

FIG. 1. (color online). Ground state energy per particle $e$ as a function of interaction strength $U / t$ at $n=0.25$ (left) and $n=0.0625$ (right). Symbols represent $e_{C P M C}$. Dashed (blue) line corresponds to the energy of a saturated ferromagnetic state $\left(e_{F M}\right) . e_{M F}$ energy is represented by the thick solid (green) line. $e_{P}$ (perturbation theory [26]) is plotted by dotdashed line.

$n=0.25$, MF predicts a paramagnetic to ferromagnetic phase transition at $U=13.9 t$. This is to be compared to the corresponding transition point $k_{F} a \sim \pi / 2$ in the continuum Stoner Hamiltonian, where $k_{F}=\left(3 \pi^{2} n\right)^{1 / 3}$ is the Fermi wave vector, and $a$ is the scattering length in continuum. When the system density is lowered to $n=0.0625$, the MF transition in the Hubbard model is at a larger interaction, $U=29.3 t$. The equation of state has also been obtained from perturbation theory for an unpolarized system [26]: $e_{P}(U, n)=e_{M F}(U, n)+$ $e_{c}(U, n)$. The last term, $e_{c}(U, n)$, is the correlation energy estimated to $\mathcal{O}\left(U^{2}\right)$. The result is also included in Fig. 1.

The CPMC result for the ground state energy $e_{C P M C}$ is obtained by averaging over twist-angles. The energies calculated from different lattice sizes are shown by different symbols in Fig. 1. It can be seen that our remaining finite-size errors are negligible on this scale. Free-electron trial wave functions are used for the constraint. In a few cases we have also checked with unrestricted HartreeFock trial wave functions, which gave statistically indistinguishable CPMC energies. The energies shown are for finite time steps, with $\Delta \tau$ satisfying $U \Delta \tau<0.2$. The residual Trotter error is $\mathcal{O}\left(10^{-2}\right)$, smaller than the symbol size.

We see that MF theory, which gives a reasonable estimate of the energy at small $U$, quickly shows severe deviations as the interaction becomes stronger. The perturbation result, $e_{P}(U, n)$, gives an improved estimate of energy for small $U$, but deviates once the system enters the intermediate interaction regime $U \gtrsim 5 t$. At the MF transition point, the CPMC energy is significantly lower than $e_{F M}$. Indeed the CPMC energy remains lower than $e_{F M}$ across the entire range of $U$ simulated. No indication of a ferromagnetic transition is seen.

Individual components of the energy are shown in Fig. 2. As $U$ increases, electrons in the unpolarized system occupy higher momentum states, outside the Fermi level, which increases the kinetic energy compared to the 

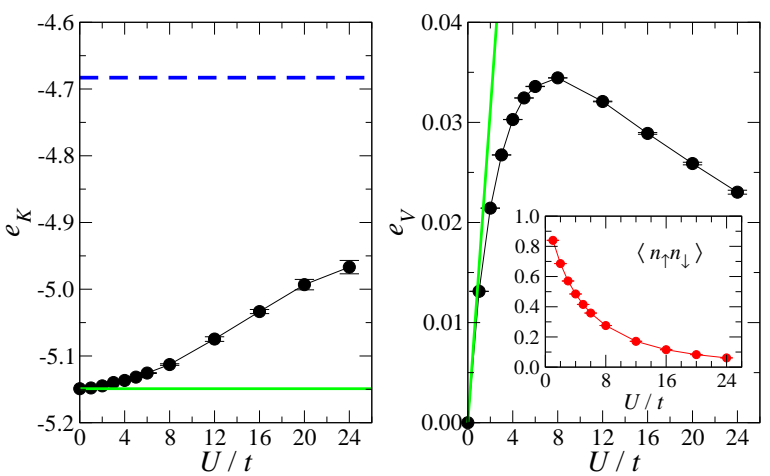

FIG. 2. (color online). Kinetic (left panel) and interaction (right panel) energies as a function of interaction strength at density $n=0.0625$. Symbols are the CPMC data obtained on an $8^{3}$ lattice. Lines are defined in the same way as in Fig. 1 The inset on the right shows the double occupancy, normalized to 1 at $U=0$.

MF result. This enables the system to drastically decrease the interaction energy, by suppressing double occupancy. The net effect is that the total energy is greatly reduced and remains below $e_{M F}$ and $e_{F M}$.

Correlation function - To probe the nature of the ground state, we examine the spin-dependent pair correlation function:

$$
g_{\sigma \sigma^{\prime}}(\mathbf{r})=\frac{1}{\bar{n}_{\sigma} \bar{n}_{\sigma^{\prime}}} \frac{1}{N} \sum_{\mathbf{r}^{\prime}}\left\langle n_{\mathbf{r}+\mathbf{r}^{\prime}, \sigma} \cdot n_{\mathbf{r}^{\prime}, \sigma^{\prime}}\right\rangle .
$$

The CPMC expectations are evaluated by the backpropagation technique [16, 27]. We average over different r's to obtain $g_{\sigma \sigma^{\prime}}(r)$, with $r \equiv|\mathbf{r}|$ since the correlation function is primarily a function of distance in the paramagnetic or ferromagnetic phases.

The anti-parallel pair correlation $g_{\uparrow \downarrow}(r)$ is a constant in a non-interacting system or in the MF solution. In the presence of interaction, a correlation hole is created surrounding each electron. At $n=0.0625$, the size of the correlation hole is $r_{\text {cor }} \lesssim \sqrt{3}$. As $U$ is increased, the correlation hole becomes deeper, as illustrated in the left panel in Fig. 3. Compared to $g_{\uparrow \downarrow}(r)$, the change in the parallel-spin pair correlation $g_{\uparrow \uparrow}(r)$ is less dramatic from the MF or non-interacting result. Strong interaction does appear to increase $g_{\uparrow \uparrow}(r)$ slightly at short distance. However, the correlation remains much less than that in the FM case.

Momentum distribution - The creation of correlation hole is a result of minimizing the interaction energy. Electrons of opposite spins rearrange their relative positions to reduce the potential energy. The cost of the rearrangement is the kinetic energy increase, as discussed earlier. This can also be observed in Fig. 4 where the
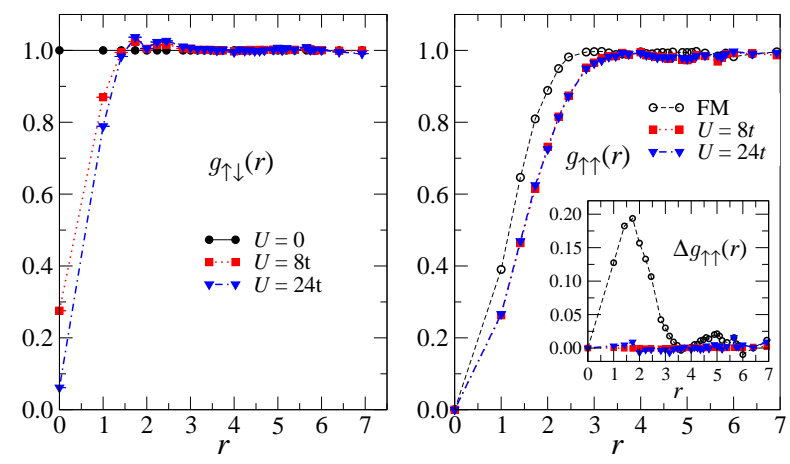

FIG. 3. (color online). Left: Anti-parallel spin-spin pair correlation function of an unpolarized state at different interaction strengths. Right: Comparison of the parallel pair correlation functions of the fully polarized state (FM) and the unpolarized states at different interaction strengths. The inset shows $\Delta g_{\uparrow \uparrow}(r)=g_{\uparrow \uparrow}(r)-g_{\uparrow \uparrow}^{0}(r)$, where $g_{\uparrow \uparrow}^{0}(r)$ is the correlation function of the unpolarized non-interacting system. In both panels, the system is an $8^{3}$ lattice at density $n=0.0625$.

momentum distribution $n_{\mathbf{k}}$ is shown for different interaction strengths. We have plotted $n_{\mathbf{k}}$ as a function of the single-particle energy level $\varepsilon(\mathbf{k})=2 \sum_{\alpha=x, y, z}[1-$ $\left.\cos \left(k_{\alpha}+\Theta_{\alpha} / L\right)\right]$, in units of the Fermi energy $\varepsilon_{F}$. Each curve contains the result of $n_{\mathbf{k}}$ from multiple $\Theta$-points. At $U=4 t$, the distribution is very close to the noninteracting momentum distribution with only a few low lying excitations near the Fermi surface (FS). As $U$ is increased, more higher $\mathbf{k}$ states are populated outside the FS. In $n_{\mathbf{k}}$ a jump appears at $\varepsilon_{F}$ which can be read off directly in our finite size simulations. The jump indicates that the system is a normal Fermi liquid, with the value of the jump proportional to the renormalization factor $Z$. Its precise value can be determined with more extensive calculations and finite size scaling.

Discussion - Although we have focused on the ground state of a homogeneous Fermi gas, it is not difficult to extend the results to the case with an external trap. For example, the kinetic energy results in Fig. 2]indicate that, with a trap, there would be a minimum in the curve of $e_{K}$ versus interaction strength, as observed in the experiment [8] (see also discussion in Ref. [9]). Effects of confinement on the kinetic energy have been investigated in detail by CPMC for trapped Bose gases [15]. The MF kinetic energy was shown to decrease monotonically because the gas expands in the trap as the scattering length $a$ is increased; on the other hand, correlation effects lead to an increase of $e_{K}$, similar to Fig. 2 This competition results in a non-monotonic curve, with a minimum in the kinetic energy at a finite scattering length.

The Hubbard model, of interest in its own right, contains some of the same features (namely itinerant electrons and local interaction) as the continuum Stoner Hamiltonian. However, there are differences with respect 

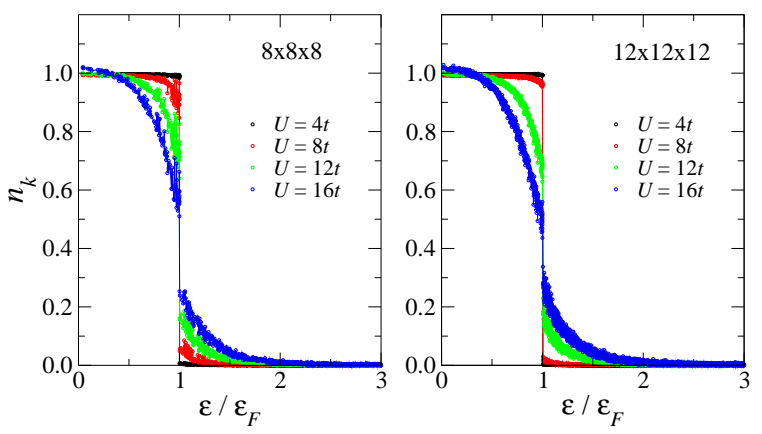

FIG. 4. The momentum distribution $n_{\mathbf{k}}$ for values of $U$ plotted as a function of the single particle energy. Two lattice sizes are shown, at the density $n=0.0625$. In each system, we average over 10 random twist angles.

to the experiment worth emphasizing. The experiment is in the continuum, using an attractive (negative) interaction with an effective positive scattering length for the excited state that describes the prepared state. (However, there are questions whether such an effective description is appropriate [12].) In our simulation, we use a discretized representation, with positive on-site interaction. As mentioned before, the lattice model leads to a scattering length bounded by roughly the lattice spacing. Using the above values for the maximum scattering length and $k_{F}$ in the unpolarized phase, we find that $k_{F} a<1.03 n^{1 / 3}$. (For the transition to the ferromagnetic phase, $n \leq 1$ since one cannot have two like-spin fermions on a single site. To focus on the dilute limit, we have done calculations for up to $n=0.5$.)

Recently the problem of itinerant ferromagnetism in repulsive Fermi gases has been studied by several groups [10, 11, 24] using the DMC method with the fixed-node approximation. These calculations all found the existence of a ferromagnetic instability. The DMC calculations were all done in the continuum, while the present calculation is for the Hubbard (lattice) model. In the DMC simulations, the atomic interaction is modeled by a repulsive potential whose range is determined by the scattering length. We note that since the scattering length diverges near resonance, the range of potential (or the range of the node in the Jastrow when a negative interaction is used) can become very large. Note that the hardsphere-like interaction is only between unlike spins. As the scattering length approaches the interparticle spacing, there is a strong tendency to separate into a spin-up and spin-down domains, to lower the interaction energy; i.e. it favors ferromagnetism.

Of lesser importance, the DMC fixed-node errors in the calculated ground state energies bias the result in favor of ferromagnetism, since nodal surfaces for the ferromagnetic state are more accurate than the spin unpolarized state [28]. Although the constrained path error from our calculations could also be biased, previous calculations indicate [16, 17, 21] that the systematic error in CPMC is smaller than the fixed-node error from single determinant trial wave functions used in these calculations.

Summary - We have examined the magnetic properties in the ground state of the dilute 3D Hubbard model, using the CPMC method and twist-averaged boundary conditions. Our simulation results indicate that there is no ferromagnetic instability in this model with strong on-site repulsions for densities up to 0.5. The ground state appears to be a paramagnetic Fermi liquid. The total energy is effectively lowered by electron correlation which, while increasing the kinetic energy, can strongly suppress double occupancy to lower the interaction energy. In the presence of a trap, the kinetic energy can be decreased by the expansion of the gas due to repulsive interaction. A kinetic energy minimum, which was observed in the experiment, can be understood in terms of the competitions between these effects. We have also discussed the difference between our calculations and recent results from DMC simulations, as well as connections and differences with the Fermi gas itinerant ferromagnetism experiments.

Acknowledgments - C.C. and S.Z. acknowledge support from ARO (56693-PH) and NSF (DMR-0535592) and D.M.C. from the OLE program. Computations were carried out at ORNL (Jaguar XT4) and William \& Mary (CPD and SciClone clusters). We thank Jie Xu for help and T.L. Ho for useful discussions.
[1] J. Hubbard, Proc. R. Soc. London Ser. A, 276, 238 (1963).

[2] Y. Nagaoka, Phys. Rev., 147, 392 (1966).

[3] D. J. Thouless, Proc. Phys. Soc. London, 86, 893 (1965).

[4] M. Takahashi, J. Phys. Soc. Japan, 51, 3475 (1982); B. Doucot and X. G. Wen, Phys. Rev. B, 40, 2719 (1989); X. Y. Zhang et al., Phys. Rev. Lett., 66, 1236 (1991); W. Putikka et al., ibid., 69, 2288 (1992).

[5] J. A. Riera and A. P. Young, Phys. Rev. B, 40, 5285 (1989); A. Barbieri et al., ibid., 41, 11697 (1990).

[6] F. Becca and S. Sorella, Phys. Rev. Lett., 86, 3396 (2001).

[7] G. Carleo et al., arxiv:1007.4260.
[8] G.-B. Jo et al., Science, 325, 1521 (2009).

[9] H. Zhai, Phys. Rev. A, 80, 051605R (2009); X. Cui and H. Zhai, ibid., 81, 041602R (2010).

[10] S. Pilati et al., arxiv:1004.1169.

[11] S.-Y. Chang et al., arxiv:1004.2680.

[12] D. Pekker et al., arxiv:1005.2366.

[13] H. Tasaki, Prog. Theor. Phys., 99, 489 (1998).

14] Y. Castin, J. Phys. IV France, 116, 89 (2004).

[15] W. Purwanto and S. Zhang, Phys. Rev. A, 72, 053610 (2005).

[16] S. Zhang et al., Phys. Rev. Lett., 74, 3652 (1995); Phys. Rev. B, 55, 7464 (1997).

[17] S. Zhang and H. Krakauer, Phys. Rev. Lett., 90, 136401 
(2003).

[18] C.-C. Chang and S. Zhang, Phys. Rev. B, 78, 165101 (2008).

[19] J. E. Hirsch, Phys. Rev. B, 31, 4403 (1985).

[20] W. A. Al-Saidi et al., J. Chem. Phys., 124, 224101 (2006); 127, 144101 (2007).

[21] W. Purwanto et al., Phys. Rev. B, 80, 214116 (2009).

[22] D. M. Ceperley and B. J. Alder, Phys. Rev. Lett., 45, 566 (1980).

[23] W. M. C. Foulkes et al., Rev. Mod. Phys., 73, 33 (2001).
[24] G. J. Conduit et al., Phys. Rev. Lett., 103, 207201 (2009).

[25] C. Lin et al., Phys. Rev. E., 64, 016702 (2001).

[26] W. Metzner and D. Vollhardt, Phys. Rev. B, 39, 4462 (1989).

[27] W. Purwanto and S. Zhang, Phys. Rev. E, 70, 056702 (2004).

[28] F. H. Zong et al., Mol. Phys., 101, 1705 (2003). 